\title{
Une exploration de la motivation en grammaire du français d'élèves du secondaire québécois
}

\author{
Isabelle Gauvin ${ }^{1}$ \\ Joël Thibeault ${ }^{2}$ \\ Carla Barroso da Costa ${ }^{1}$ \\ Isabelle Plante ${ }^{1}$ \\ Valérie Bélanger ${ }^{3}$ \\ ${ }^{1}$ Université du Québec à Montréal, Montréal, Canada \\ ${ }^{2}$ Université d'Ottawa, Ottawa, Canada \\ ${ }^{3}$ Collège Reine-Marie, Montréal, Canada
}

\section{Pour citer cet article :}

Gauvin, I., Thibeault, J., Barroso da Costa, C., Plante, I., \& Bélanger, V. (2021). Une exploration de la motivation en grammaire du français d'élèves du secondaire québécois. Didactique, 2(1), 40-54.

Résumé : La recherche examine la motivation d'élèves à l'égard de la grammaire selon deux composantes : les attentes de succès et la valeur accordée à la grammaire. Ainsi, 58 élèves québécois de $2 \mathrm{e}$ secondaire ont répondu à un questionnaire évaluant leur motivation en grammaire. Puis, les différences selon le genre et le rendement antérieur en grammaire et en écriture ont été examinées. Les résultats révèlent une corrélation entre les deux composantes de la motivation. De plus, ces deux composantes diffèrent selon le rendement en grammaire et en écriture des élèves, mais pas selon le genre. Ces résultats orientent la recherche et l'intervention futures pour motiver les élèves en grammaire et, ultimement, favoriser le développement de leur compétence en écriture.

Mots-clés : motivation, grammaire, apprentissage, didactique, français langue d'enseignement 


\section{Introduction}

« Aujourd'hui, commençons la journée par une capsule de grammaire!»: quel.le enseignant.e de français au secondaire n'a jamais été témoin de réactions fortes, la plupart du temps négatives, après une telle déclaration devant sa classe? La scène est si fréquente qu'elle en est presque devenue un cliché, donnant l'impression tenace à d'innombrables enseignant.e.s de français au secondaire que leurs élèves n'aiment pas la grammaire. Mais qu'en est-il vraiment? En réalité, la motivation de l'élève à apprendre un savoir peut difficilement être observée objectivement à partir de son seul comportement en classe.

La disposition des enseignants envers la grammaire, quant à elle, semble plutôt favorable. En effet, une recherche récente portant sur le rapport $\grave{a}^{1}$ d'enseignant.e.s de français en formation au Québec à propos de leur propre apprentissage et de leur futur enseignement de la grammaire établit un portrait non équivoque: les futur.e.s enseignant.e.s questionné.e.s sont très positif.ve.s à l'égard de la grammaire, si bien qu'ils.elles entrevoient très positivement leurs futures pratiques enseignantes, beaucoup plus positivement par ailleurs que leur propre expérience d'apprentissage de la grammaire (Gauvin, Lemay et Aubertin, 2016). Si les futur.e.s enseignant.e.s éprouvent d'excellentes dispositions à l'égard de la grammaire, qu'en est-il de celles des élèves? Leur expérience de l'apprentissage grammaticale est-elle positive? En d'autres mots, sont-ils motivés à apprendre la grammaire du français?

Comme le souligne Boscolo (2012), la motivation est un construit théorique utilisé afin de rendre compte de l'activation, de l'intensité, de la persistance et de la qualité d'un comportement. Il s'agit sans surprise d'une variable dont on doit tenir compte pour comprendre ce qui sous-tend la réussite scolaire (Plante, O'Keefe et Théorêt, 2013; Steinmayr et Spinath, 2009). Pour ces raisons, notre étude ${ }^{2}$, à visée exploratoire, a donc pour objectif général d'établir un portrait motivationnel d'élèves du secondaire en ce qui a trait à la grammaire.

\section{Cadre de référence et recension des écrits}

La motivation est un construit complexe, pluriel, qui gagne en popularité dans la recherche en éducation et qui, eu égard à l'enseignement grammatical, n'a fait l'objet que d'une quantité relativement limitée d'études. Il convient donc de définir ce qui renvoie pour nous

\footnotetext{
${ }^{1}$ Nous définissons sommairement le rapport à comme la disposition, cognitive et affective, à l'égard d'un objet de savoir, ici la grammaire et la didactique de la grammaire (Gauvin et Aubertin, 2014).

2 Étude financée par le Centre d'études sur l'apprentissage et la performance de l'Université du Québec à Montréal, le CEAP UQAM (https://ceap.uqam.ca).
} 
à la motivation et de mettre en avant les quelques études qui ont jeté un éclairage sur le profil motivationnel d'élèves en grammaire.

\section{Qu'entendons-nous par motivation ?}

Les quelques travaux qui ont abordé la motivation en la mettant en relation avec l'apprentissage de la grammaire (Boyer, Bouffard et Lebrun, 2016; Thibeault et Fleuret, 2019) reposent à notre connaissance sur certaines des composantes du modèle attentesvaleur (Fréchette-Simard, Plante, Dubeau et Duchesne, 2019; Barron et Hulleman, 2015; Schunk, Meece et Pintrich, 2014; Wigfield, Eccles, Schiefele et Roeser, 2008). Dans ce modèle, on postule que le construit «motivation" se décline en deux composantes complémentaires qui, ensemble, forment la dynamique motivationnelle de l'apprenant. La première des deux composantes, les attentes de succès, se traduit par la perception de compétence de l'apprenant, c'est-à-dire le sentiment subjectif qu'il se construit à l'égard de sa propre compétence dans une discipline donnée, ici la grammaire. La seconde composante, la valeur, renvoie quant à elle à la perception de l'apprenant.e quant à l'utilité de la discipline, à son intérêt vis-à-vis d'elle et à sa perception de l'importance qu'elle revêt.

Comme nous le disions précédemment, la recherche réalisée jusqu'à présent sur la motivation en grammaire, bien que récente et émergente, a permis de mettre au jour quelques résultats en lien avec certaines des composantes du modèle attentes-valeur. Boyer, Bouffard et Lebrun (2016) ont ciblé la composante attentes de succès du modèle en déterminant si les biais d'autoévaluation de compétence en orthographe grammaticale et le genre de l'individu étaient associés au rendement de 295 élèves de première secondaire (environ 12 ans) dans ce même domaine. À partir de l'analyse d'une dictée, d'un questionnaire évaluant les perceptions de compétence en orthographe et d'un test sur les habiletés scolaires, ces chercheuses ont noté une relation positive entre la perception de compétence en grammaire et le rendement des élèves en orthographe grammaticale. Eu égard à la variable genre, les auteures montrent que les résultats liés au rendement en orthographe grammaticale sont également supérieurs chez les filles; dans cette même perspective, elles remarquent aussi une différence significative en faveur des filles pour ce qui est de la perception de compétence en grammaire. Cependant, les résultats montrent également que, si le genre contribue au rendement de l'élève, «il perd son importance lorsque sont introduits les biais d'autoévaluation » (p. 52). Les chercheuses en concluent donc que les résultats faibles en orthographe grammaticale qui sont notés chez les garçons peuvent en partie s'expliquer par une sous-estimation de leur compétence en grammaire. 
Pour leur part, Thibeault et Fleuret (2019) ont réalisé une étude de cas multiples visant à décrire la motivation en grammaire de huit élèves plurilingues de $5^{\mathrm{e}}-6^{\mathrm{e}}$ années (environ 1011 ans) scolarisé.e.s en Ontario francophone. Pour ce faire, grâce à un questionnaire sur la motivation et à une entrevue individuelle, ils se sont intéressés à la perception de l'élève quant à l'utilité de la grammaire, à son intérêt en grammaire et à sa perception de compétence en grammaire. Ils ont aussi exploré les liens potentiels entre la motivation en grammaire des élèves et leur rendement lors de tâches écrites (activité à trous et productions écrites) sur l'accord du verbe en nombre. Les résultats ont fait émerger des tendances intéressantes. D'abord, tel que prévu, les élèves les plus performant.e.s affichent des scores motivationnels plus élevés. De plus, les données de l'étude ont montré que même si les élèves voient l'utilité de la grammaire en classe, ils manifestent apparemment un intérêt moindre par rapport à cette discipline. Étant donné le nombre limité de participant.e.s dans cette étude, ces résultats sont à considérer avec précaution, mais offrent néanmoins des pistes intéressantes pour la recherche future.

En somme, la recherche quantitative réalisée à ce jour sur la motivation en grammaire semble s'être intéressée uniquement à l'une des composantes du modèle attentes-valeur : les attentes de succès, qui sont opérationnalisées par les perceptions de compétence. Les autres composantes, quant à elles, ont également fait l'objet d'une recherche, mais cette dernière repose sur un échantillon restreint. Qui plus est, elle ne s'est pas intéressée aux relations pouvant exister entre les différentes composantes de la motivation en grammaire. De ce fait, il nous parait important de comprendre, en nous appuyant sur la recherche antérieure, les relations qu'entretiennent ces différentes composantes, et ce, en les examinant selon le genre des élèves et leur niveau de performance. Si l'ensemble de ces variables a été étudié par des chercheur.seuse.s avant nous, aucune étude adoptant un devis quantitatif n'a tenté de brosser un portrait holistique de la motivation en grammaire des élèves. Pourtant, un tel portrait pourrait permettre de proposer des pratiques didactiques qui prennent davantage en compte la motivation des élèves. À l'heure actuelle, l'état de la recherche en didactique de la grammaire ne permet pas d'identifier les pratiques les plus susceptibles de soutenir ou de freiner leur motivation.

\section{Objectifs spécifiques de recherche}

Afin d'établir le portrait motivationnel d'élèves du secondaire au Québec en grammaire, notre étude vise à répondre à deux objectifs :

1. Décrire la motivation des élèves en grammaire telle qu'elle se manifeste dans leur perception de compétence en grammaire et la valeur (intérêt, importance et utilité) qu'ils.elles lui accordent; 
2. Décrire la motivation des élèves en grammaire en fonction des variables indépendantes suivantes :

a. Leur genre (garçons ou filles)

b. Leur rendement en grammaire et en écriture (faible, moyen, élevé)

\section{Méthodologie}

L'étude dont nous présentons les résultats est de nature exploratoire. En effet, bien que la recherche actuelle nous offre des pistes intéressantes pour comprendre la motivation en grammaire des élèves, les données disponibles ne permettent pas encore une prise en considération holistique des composantes du modèle attentes-valeur pour mieux capter les différences motivationnelles selon le genre et le niveau de rendement antérieur.

\section{L'échantillon}

La recherche a été effectuée au sein de trois classes de 2e secondaire (environ 14 ans) d'une école privée de Montréal. Le recrutement a été effectué par l'enseignante qui a accepté de participer à la recherche. Comme il s'agit d'un échantillon de convenance, les résultats ne pourraient prétendre à une généralisation.

Ainsi avons-nous pu recruter 58 participant.e.s, 34 filles et 24 garçons issus de trois classes au sein desquelles un enseignement régulier du français langue d'enseignement était dispensé (ministère de l'Éducation, 2011), et ce, par la même enseignante. Il existait donc une harmonisation dans les pratiques que les élèves ont vécu en enseignement grammatical. En accord avec les directives du Comité éthique de l'Université du Québec à Montréal, tous les élèves qui ont participé à l'étude ont obtenu le consentement écrit d'un de leurs parents.

\section{La collecte et l'analyse de données}

Pour dresser le portrait motivationnel des élèves, nous avons utilisé un questionnaire comprenant 12 items (cf. annexe). Pour chacun de ces items, les élèves devaient répondre sur une échelle de Likert allant de 1 à 7,7 correspondant à une plus forte motivation en grammaire. Le temps moyen pour remplir le questionnaire était d'environ cinq minutes. Ce questionnaire, qui a initialement été élaboré en anglais pour mesurer la motivation à l'égard de la lecture (Eccles et Wigfield, 1995), a été adapté en français et validé auprès d'élèves québécois (Plante et collab., 2013). Aux fins de la présente étude, ce questionnaire a été adapté pour cibler la grammaire. Par exemple, l'item « Comment crois-tu réussir cette 
année en lecture ?» a été remplacé par l'item «Comment crois-tu réussir cette année en grammaire?».

Pour examiner la structure factorielle des données et ainsi vérifier si les items qui sont conceptuellement liés produisent des patrons de réponse similaires, une analyse en composantes principales a été menée. Pour l'interprétation des composantes, la technique de rotation non orthogonale oblimin a été retenue, puisqu'elle est recommandée lorsque les facteurs (ou échelles de mesure) sont liés les uns aux autres (Tabachnick et Fidell, 2013), comme c'est le cas des attentes de succès et des composantes de la valeur.

Les résultats des analyses ont montré que les items se divisaient en deux composantes : (1) la perception de compétence en grammaire (items $1,3,6,8,11$ ) et la valeur attribuée à la grammaire (items 2, 4, 5, 7, $9,10,12$ ). Subséquemment, des analyses de fiabilité ont été menées pour vérifier le degré de consistance interne des réponses issues des différents items qui sont inclus dans chacune de ces deux composantes, tel que reflété dans l'indicateur alpha de Cronbach. Le tableau 3.1 présente la moyenne et l'écart-type pour chacun de ces items, ainsi que l'indicateur alpha de Cronbach pour chaque composante.

Tableau 3.1 : Composantes de la motivation en grammaire

\begin{tabular}{|c|c|c|c|c|}
\hline Composante & Item & $\begin{array}{l}\text { Moye } \\
\text { (écart- }\end{array}$ & & $\begin{array}{l}\text { Alpha de } \\
\text { Cronbach }\end{array}$ \\
\hline \multirow{5}{*}{ 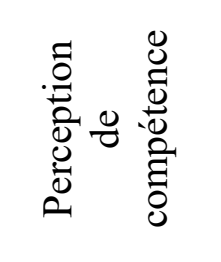 } & 1 & $4,61(1,20)$ & \multirow{5}{*}{$\begin{array}{c}4,32 \\
(1,05)\end{array}$} & \multirow{5}{*}{0,91} \\
\hline & 3 & $4,01(1,26)$ & & \\
\hline & 6 & $4,11(1,26)$ & & \\
\hline & 8 & $4,72(1,15)$ & & \\
\hline & 11 & $4,24(1,20)$ & & \\
\hline \multirow{7}{*}{ 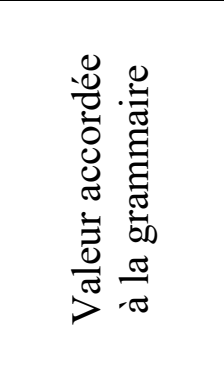 } & 2 & $3,98(1,61)$ & \multirow{7}{*}{$\begin{array}{c}3,96 \\
(1,16)\end{array}$} & \multirow{7}{*}{0,86} \\
\hline & 4 & $5,45(1,32)$ & & \\
\hline & 5 & $2,80(1,34)$ & & \\
\hline & 7 & $2,47(1,47)$ & & \\
\hline & 9 & $3,91(1,79)$ & & \\
\hline & 10 & $4,67(1,64)$ & & \\
\hline & 12 & $4,65(1,63)$ & & \\
\hline
\end{tabular}

L'analyse en composantes principales met en évidence une consistance interne élevée, avec des valeurs supérieures à ,70, seuil qui est généralement retenu (Nunally, 1978).

L'étude a utilisé des tests $t$ pour des échantillons appariés visant la comparaison des moyennes des deux dimensions de la motivation : la perception de compétence et la valeur 
attribuée à la grammaire. Le test $t$ pour des échantillons indépendants a été également réalisé afin de comparer les moyennes des garçons et des filles pour les deux dimensions de la motivation. De plus, l'analyse de corrélation a été effectuée pour vérifier l'existence d'un lien entre les deux composants de la motivation en grammaire. Finalement, des analyses de variance multiple (MANOVA) ont permis d'examiner si la perception de compétence et la valeur que les étudiants accordent à la grammaire différaient selon le niveau de performance en grammaire et en écriture. Toutes les analyses ont été réalisées en utilisant le logiciel SPSS, version 24 (IBM-Corporation, 2016).

\section{Résultats}

La présente section présente les résultats concernant la motivation des élèves en grammaire selon (4.1) leur perception de compétence en grammaire et la valeur qu'ils lui accordent, (4.2) le genre, ainsi que (4.3) le niveau de performance des élèves en grammaire et en écriture.

\section{Motivation des élèves en grammaire selon deux composantes}

Sur le plan descriptif ( $c f$. tableau 1 plus haut dans le texte), il est possible de constater que, parmi les moyennes des items de la composante « perception de compétence », l'item 3 ( $\mathrm{Si}$ tu devais classer les élèves de la classe en fonction de leur rendement en grammaire, du moins bon au meilleur, où te situerais-tu?) présente la moyenne la plus faible $(\mathrm{m}=4,01$, é.t. $=1,26)$, tandis que l'item 8 (Comment crois-tu réussir cette année en grammaire?) indique la moyenne la plus élevée $(\mathrm{m}=4,72$, é.t. $=1,15)$.

En ce qui concerne la composante « valeur accordée à la grammaire », l'item 7 (Jusqu'à quel point aimes-tu faire des activités de grammaire?) est celui avec la moyenne la plus faible $(\mathrm{m}=2,47$, é.t. $=1,47)$, tandis que l'item 4 (Jusqu'à quel point est-il important pour toi d'obtenir de bonnes notes en grammaire?) indique la moyenne la plus élevée $(\mathrm{m}=$ $5,45$, é.t. $=1,32)$.

Sur le plan des analyses inférentielles, le test $t$ pour échantillons appariés indique une différence statistiquement significative entre les moyennes des deux composantes de la motivation $(\mathrm{p}=0,04)$. Ainsi, la perception de compétence en grammaire des élèves $(\mathrm{m}=$ $4,32, \mathrm{sd}=1,05)$ est plus élevée que la valeur qu'ils accordent à la grammaire $(\mathrm{m}=3,96, \mathrm{sd}$ $=1,16)$. 
Par ailleurs, les résultats d'une corrélation de Pearson entre la perception de compétence en grammaire et la valeur accordée à la grammaire montrent que plus l'élève se sent compétent en grammaire, plus il lui accorde de la valeur $\left(r=0,340^{* *} ; p=0,010\right)$.

\section{Motivation des élèves en grammaire selon le genre}

Nous avons aussi analysé la variabilité de la perception de compétence en grammaire et de la valeur accordée par les élèves à la grammaire selon leur genre. Les résultats d'un test $t$ à deux échantillons indépendants montrent que la perception de compétence en grammaire ne diffère pas significativement entre les filles et les garçons $\left(t_{(56)}=-1,911, p=0,061\right)$. Le résultat est semblable pour la valeur accordée à la grammaire $\left(\mathrm{t}_{(56)}=-0,940, \mathrm{p}=0,351\right)$. Autrement dit, la perception de compétence ainsi que la valeur accordée à la grammaire ne varient pas significativement en fonction du genre.

\section{Motivation des élèves en grammaire selon leur performance en grammaire et en écriture}

Les performances des élèves en grammaire et en écriture ont été établies à partir d'une analyse par quartiles de leurs résultats provenant de deux sources : la note obtenue à un examen de grammaire complété en fin d'année scolaire et la note en écriture inscrite sur le sommaire de la dernière période d'évaluation de l'année. Ces résultats ont été colligés en juin, soit environ 4 mois après la passation du questionnaire.

Le premier quartile compose le niveau faible, les deuxième et troisième quartiles, le niveau moyen et le quatrième, le niveau fort. Le tableau 4.1 présente le nombre de participants par niveau de performance en grammaire et en écriture.

Tableau 4.1 : Nombre de participants par niveau de performance en grammaire et en écriture

\begin{tabular}{ccc}
\hline & $\begin{array}{c}\text { Niveau de } \\
\text { performance }\end{array}$ & $\mathrm{N}$ \\
\hline \hline \multirow{3}{*}{ Grammaire } & Faible & 14 \\
& Moyen & 26 \\
& Fort & 16 \\
\hline \multirow{2}{*}{ Écriture } & Faible & 14 \\
& Moyen & 26 \\
& Fort & 16 \\
\hline
\end{tabular}


L'analyse descriptive de la motivation selon la performance des élèves en grammaire ( $c f$. figure 1 plus bas) indique que le groupe classifié comme faible en grammaire présente également des moyennes plus faibles dans les deux composants de la motivation ( $\mathrm{m}$ Perception de compétence $=3,73$, é.t. $=0,78 ; \mathrm{m}$ Valeur accordée à la grammaire $=3,70$, é.t. $=1,26$ ). De son côté, le groupe considéré fort en grammaire obtient des moyennes plus élevées dans les deux composantes de la motivation ( $\mathrm{m}$ Perception de compétence $=4,67$, é.t. $=1,16 ; \mathrm{m}$ Valeur accordée à la grammaire $=4,45$, é.t $=0,70$ ). Le résultat du test MANOVA montre un effet statistiquement significatif du rendement en grammaire sur les deux composantes de la motivation $\left(\mathrm{F}_{(2,53)}\right.$ $=3,77, p=0,03)$, avec une taille d'effet modérée $\left(\eta^{2}=0,12\right)$. L'analyse de comparaisons multiples Bonferroni indique une différence significative dans la motivation pour les élèves forts et faibles en grammaire $(\mathrm{p}=0,029)$. Autrement dit, les élèves dont la performance en grammaire est forte $(\mathrm{m}=4,67$, = é.t. 1,16$)$ sont ceux qui ont une perception de compétence en grammaire plus élevée ; les élèves dont la performance en grammaire est considérée plus faible $(\mathrm{m}=3,73$, é.t. $=0,68)$ rapportent une perception de compétence moindre. Par contre, aucune différence statistiquement significative sur la perception de compétence n'a été observée entre les élèves considérés moyens en grammaire $(m=4,41$, é.t. $=0,94)$ et ceux qui sont considérés forts $(\mathrm{p}=0,984)$, de même que chez les élèves moyens par rapport à ceux qui sont faibles $(\mathrm{p}=0,144)$.

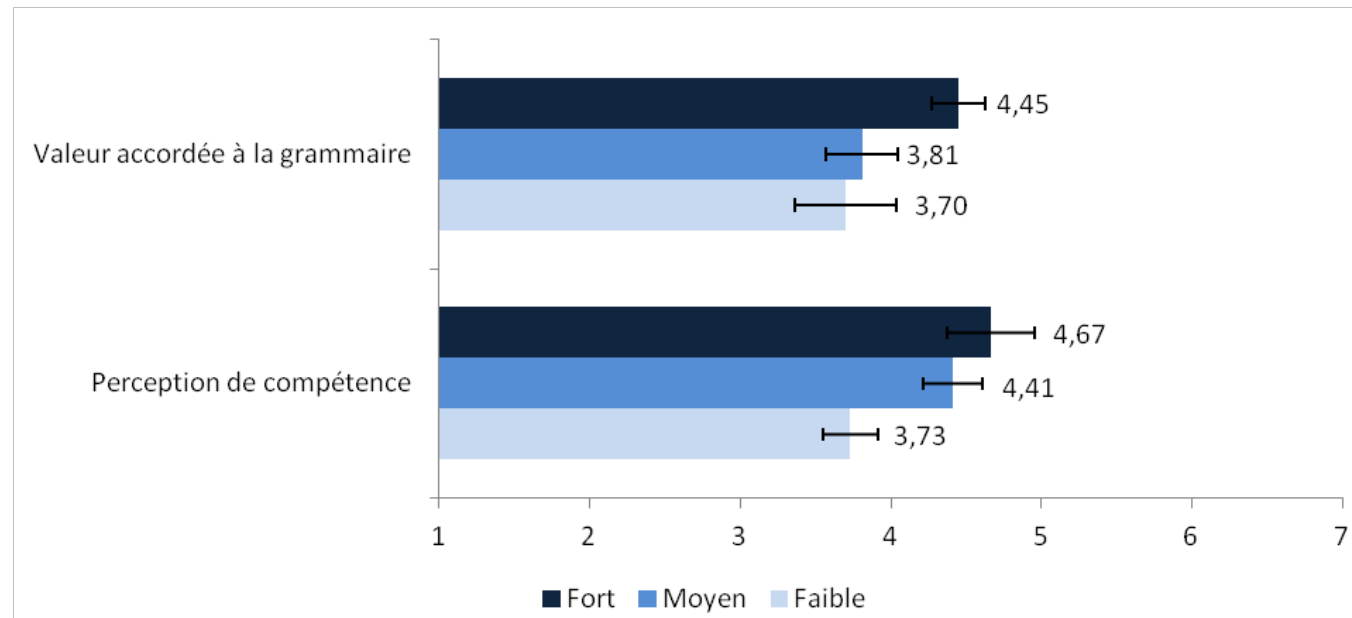

Figure 4.1: Moyenne par composante de la motivation en grammaire selon les performances des élèves en grammaire

La motivation selon la performance des élèves en écriture ( $c f$. figure 2 plus bas), qui suit une tendance similaire à leur performance en grammaire, indique que le groupe classifié comme faible en écriture présente également des moyennes plus faibles dans les deux composants de la motivation en grammaire ( $m_{\text {Perception de compétence }}=3,82$, é.t. $=0,82 ;$ mValeur accordée à la grammaire $=3,59$, é.t $=1,10)$. Le groupe considéré plus fort en écriture, quant à lui, 
obtient des moyennes plus élevées dans les deux composantes de la motivation en grammaire (mPerception de compétence $=4,84$, é. $=0,96 ; m_{V a l e u r}$ accordée à la grammaire $=4,46$, é.t $=$ 0,98). Le résultat du test MANOVA indique un effet statistiquement significatif du rendement en écriture sur les deux composantes de la motivation confondues $\left(\mathrm{F}_{(2,53)}=\right.$ $4,582, p=0,015)$, avec une taille d'effet modérée $\left(\eta^{2}=0,147\right)$. Les résultats des analyses de comparaisons multiples Bonferroni mettent en évidence une différence significative de motivation en grammaire entre les élèves faibles et forts $(p=0,014)$, mais pas entre les élèves forts et moyens ( $p=0,118)$, ni moyens et faibles $(p=0,652)$.

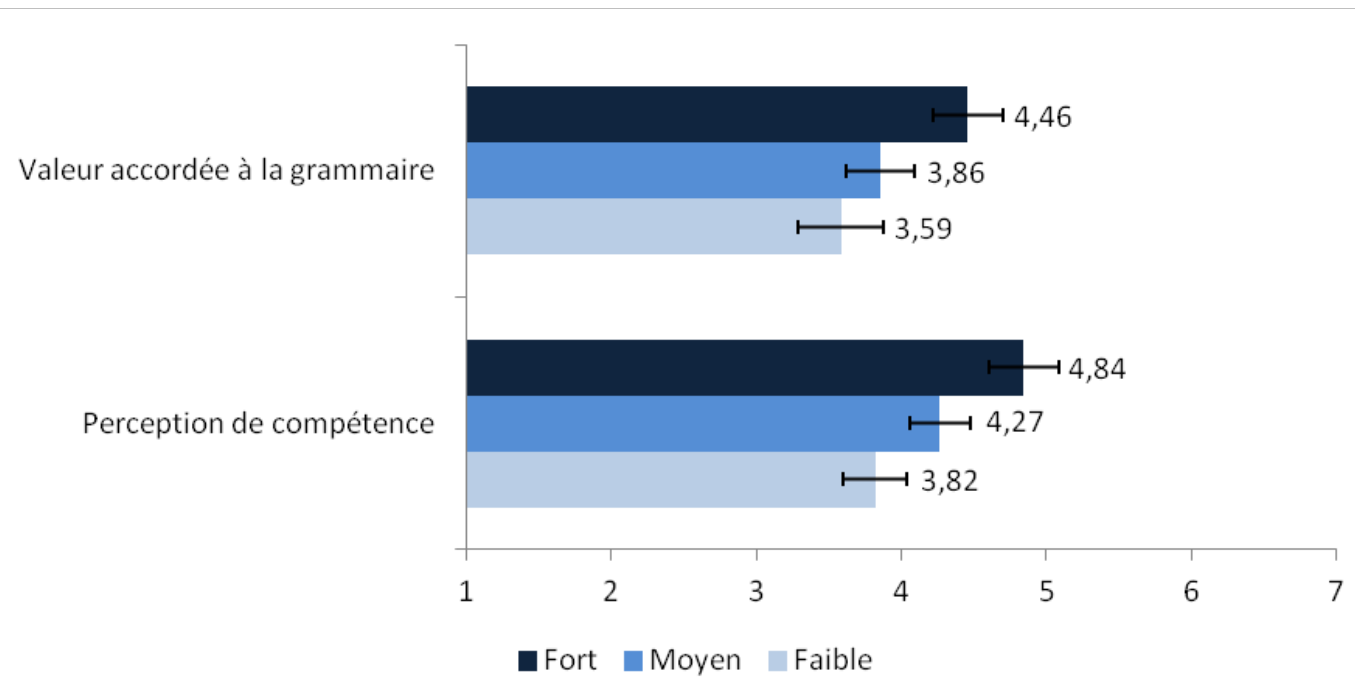

Figure 4.2: Moyenne par composante de la motivation en grammaire selon les performances des élèves en écriture

\section{Discussion et conclusion}

L'analyse des réponses de 58 élèves de $2^{\mathrm{e}}$ secondaire à notre questionnaire nous a permis de brosser un premier tableau de leur profil motivationnel en grammaire et de répondre à nos deux objectifs: (1) décrire la motivation des élèves en grammaire telle qu'elle se manifeste dans leur perception de compétence en grammaire et la valeur (intérêt, importance et utilité) qu'ils.elles lui accordent ( $c f$. section 4.1) et (2) décrire la motivation des élèves en grammaire en fonction (a) de leur genre ( $c f$. section 4.2), et (b) de leur niveau de rendement en grammaire et en écriture ( $c f$. section 4.3).

La première composante motivationnelle que nous avons évaluée, la perception de la compétence à l'égard de la grammaire, nous indique que les élèves sont tout en nuances à cet égard : ils.elles ne sont pas particulièrement confiant.e.s, mais ils démontrent tout de même une certaine assurance. En comparaison, les résultats aux items relevant de la 
deuxième composante laissent l'impression qu'ils.elles sont moins convaincu.e.s de la valeur à accorder à la grammaire. Fait intéressant, les élèves qui tendent à être plus confiant.e.s en leur compétence en grammaire y accordent généralement une plus grande valeur. Ce constat est d'ailleurs renforcé par le fait que les élèves qui valorisent le plus la grammaire et qui se sentent les plus compétent.e.s dans cette discipline, c'est-à-dire les élèves les plus performant.e.s en grammaire et en écriture sont ceux qui sont les plus motivé.e.s.

Ces observations rejoignent celles d'études antérieures sur la motivation en grammaire d'élèves un peu plus jeunes. En effet, il a déjà été constaté que des élèves plurilingues du $3^{\text {e }}$ cycle du primaire perçoivent l'utilité de la grammaire en classe, et ce, même si leur intérêt n'est pourtant pas aussi grand pour cette discipline (Thibeault et Fleuret, 2019). De plus, l'effet du rendement en grammaire (Thibeault et Fleuret, 2019) et en orthographe grammaticale (Boyer et collab., 2016) sur la perception de compétence a déjà été observée. Toujours selon les données recueillis par questionnaire auprès de nos élèves, il semble que les deux indicateurs motivationnels en grammaire ne varient pas selon le genre. Ce résultat est plutôt surprenant considérant les écrits disponibles sur le sujet. Par exemple, l'étude de Boyer, Bouffard et Lebrun (2016) a montré que les filles rapportaient une perception de compétence plus élevée que les garçons en grammaire. En outre, la maîtrise de la grammaire est directement liée à la réussite en français, un domaine où les filles sont typiquement plus motivées (ex. Plante, De la Sablonnière, Aronson et Théorêt, 2013) et réussissent mieux (pour une méta-analyse, voir Voyer et Voyer, 2014). Bien qu'il soit possible que l'absence de résultat significatif s'explique par un manque de puissance statistique, ces résultats soulignent la nécessité d'explorer davantage la motivation en grammaire selon le genre pour vérifier sa contribution dans la réussite scolaire des garçons et des filles en français.

Cette étude exploratoire suggère que les élèves les plus performants sont ceux qui affichent la plus grande motivation; ainsi témoigne-t-elle de l'importance de la perception de compétence en tant que composante motivationnelle et en tant que facteur de réussite dans l'apprentissage de la grammaire et de l'écriture. Ces constats permettent de proposer un premier portrait de la motivation en grammaire afin, notamment, d'identifier les aspects qui peuvent faire obstacle à son apprentissage et ceux qui, au contraire, peuvent servir de tremplin. Un tel portrait pourrait également contribuer à identifier les pratiques didactiques les plus à même de soutenir la motivation des élèves dans leur apprentissage de la grammaire. Une étude sur un plus vaste échantillon est évidemment nécessaire pour proposer un portrait plus précis, qui tendrait vers une certaine généralisation. 


\section{Références}

Barron, K. E. et Hulleman, C. S. (2015). Expectancy-Value-Cost model of motivation. Dans Wright, J. D. (dir.), International encyclopedia of the social \& behavioral sciences, 2nd edition (Vol. 8, pp. 503-509). Oxford: Elsevier Ltd. doi:10.1016/B9780-08-097086-8.26099-6

Boscolo, P. (2012). La fatica e il piacere di imparare. Psicologia della motivazione scolastica. Turin, Italie : UTET Università.

Boyer, P., Bouffard, T. et Lebrun, M. (2016). Relation entre le biais d'autoévaluation de compétence des élèves en orthographe grammaticale et leur performance. Revue des sciences de l'éducation, 42(1), 39-60. doi: https://doi.org/10.7202/1036893ar.

Eccles, J. S. et Wigfield, A. (1995). In the Mind of the Actor: The Structure of Adolescents' Achievement Task Values and Expectancy-Related Beliefs. Personnality and Social Psychology Bulletin, 21(3), 215-225. doi: 10.1177/0146167295213003.

Gauvin, I., Lemay, R. et Aubertin, P. (2016). Savoirs en grammaire et en didactique de la grammaire chez des étudiants en enseignement du français au secondaire. Rapport de recherche. Disponible en ligne: http://www.frqsc.gouv.qc.ca/partenariat/nosresultats-de-recherche/histoire?id=7lvhwe2z 1461263604964.

Gauvin, I. et Aubertin, P. (2014). Le rapport à la grammaire et à la didactique de la grammaire de futurs enseignants de français langue première au secondaire : un aperçu. Actes Congrès mondial de linguistique française, Berlin. Disponible en ligne :

https://www.research gate.net/publication/307742722 Le _rapport_a la_grammaire_et a la didactique de la_grammaire de futurs enseignants de francais langue premiere au_second aire un apercu.

Fréchette-Simard, C., Plante, I., Dubeau, A. et Duchesne, S. (2019). La motivation scolaire et ses théories actuelles : une recension théorique. Revue des sciences de l'éducation de McGill, 54(3), 500-518. Disponible en ligne: https://mje.mcgill.ca/article/view/9664.

Ministère de l'éducation (2011). Progression des apprentissages, français langue d'enseignement. Québec : Gouvernement du Québec.

Nunally, J. C. (1978). Psychometrie theory (2e éd.). New York, NY: McGrawHill.

Plante, I., O'Keefe, P. A. et Théorêt, M. (2013). The relation between achievement goal and expectancy-value theories in predicting achievement-related outcomes: A Test of four theoretical conceptions. Motivation \& Emotion, 37(1), 65-78, doi : 10.1007/s11031-012-9282-9.

Plante, I., De la Sablonnière, R., Aronson, J.M. et Théorêt, M. (2013). Gender stereotype endorsement and achievement-related outcomes : The role of competence beliefs and task values. Contemporary Educational Psychology, 38(3), 225235. http://dx.doi.org/10.1016/j.cedpsych.2013.03.004 
Thibeault, J. et Fleuret, C. (2019). Un regard qualitatif sur la motivation en grammaire d'élèves de la fin de l'ordre élémentaire scolarisés en contexte francophone minoritaire. Language and Literacy, 21(1), 19-38. doi: https://doi.org/10.20360/langandlit29335.

Schunk, D. H., Meece, J. L. et Pintrich, P. R. (2014). Motivation in education: theory, research, and applications. Upper Saddle River, NJ: Pearson Merrill Prentice Hall.

Steinmayr, R. et Spinath, B. (2009). The importance of motivation as a predictor of school achievement. Learning and Individual Differences, 19(1), 80-90.

Tabachnick, B. G., \& Fidell, L. S. (2013). Using multivariate statistics. (6th ed.). Boston: Pearson Education.

Voyer, D., \& Voyer, S. D. (2014). Gender differences in scholastic achievement: A metaanalysis.

Psychological

Bulletin,

140(4),

1174-1204. http://dx.doi.org/10.1037/a0036620.

Wigfield, A., Eccles, J. S., Schiefele, U. et Roeser, R. (2008). Development of achievement motivation. Dans Damon, W. et Lerner, R. M. (dir.), Child and adolescent development: An advanced course (pp. 406-434). New York: Wiley.Best, F. (1973). Pour une pédagogie de l'éveil. Paris : Bourrelier Éducation/A. Colin. 


\section{Annexe}

\section{Questionnaire}

Tu as la chance de m'aider à découvrir ce que tu ressens à propos des facteurs impliqués dans la réussite scolaire en grammaire. Ce n'est pas un examen. II n'y a pas de bonnes ou de mauvaises réponses et tous les élèves auront des réponses différentes. Ce questionnaire est confidentiel, ce qui signifie que nous ne dirons tes réponses à personne. Avant de commencer, essayons avec deux exemples.

EXEMPLES

\begin{tabular}{|c|c|c|c|c|}
\hline $\begin{array}{c}\text { Tout à fait en } \\
\text { désaccord }\end{array}$ & $\begin{array}{c}\text { Un peu en } \\
\text { désaccord }\end{array}$ & Neutre & Un peu d'accord & $\begin{array}{c}\text { Tout à fait } \\
\text { d'accord }\end{array}$ \\
\hline 1 & 2 & 3 & 4 & 5 \\
\hline
\end{tabular}

1. J'aime regarder la télévision.

$\begin{array}{lllll}1 & 2 & 3 & 4 & 5\end{array}$

Comprends-tu bien la phrase? Jusqu'à quel point cette phrase correspond-elle à ce que tu ressens? Encercle, sur l'échelle de 1 à 5 , le chiffre qui correspond le mieux à ce que tu ressens.

2. Je ne suis pas bon.ne en dessin.

$1 \quad 2 \quad 3 \quad 4$

45

Ma motivation scolaire en grammaire

Encercle, sur l'échelle de 1 à 7, le chiffre qui correspond le mieux à ce que tu ressens pour chacun des énoncés cidessous.

1. Depuis le début de l'année scolaire, comment as-tu réussi en grammaire ?
1
2
3
4
5
6
7

Très mal

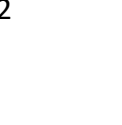

2. Jusqu'à quel point crois-tu que les exercices de grammaire te seront utiles pour la vie de tous les jours, à l'extérieur de l'école?
1
2
3
4
5
6
7
Pas du tout utiles

3. Si tu devais classer les élèves de la classe en fonction de leur rendement en grammaire (du moins bon au meilleur), où te situerais-tu?
1
2
3
4
5
6
Le meilleur
Le moins bon

4. Jusqu'à quel point est-il important pour toi d'obtenir de bonnes notes en grammaire?
1
2
3
4
5
6
7
Pas du tout important

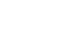

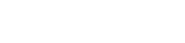

5 En général, je trouve les exercices de grammaire...
1
2
3
4
5
6
7
Très ennuyeux

(1)

(1)
Très intéressant

6. Jusqu'à quel point es-tu bon.ne en grammaire ? 


$\begin{array}{lllllrr}1 & 2 & 3 & 4 & 5 & 6 & 7 \\ \text { Pas bon.ne du tout } & & & & \text { Très bon(ne) }\end{array}$

7. Jusqu'à quel point aimes-tu faire des activités de grammaire?

$\begin{array}{lllllrr}1 & 2 & 3 & 4 & 5 & 6 & 7 \\ \text { Pas vraiment } & & & & & \text { Vraiment }\end{array}$

8. Comment crois-tu réussir cette année en grammaire ?
1
2
3
4
5
6
7
Très bien
Très mal

9. Jusqu'à quel point crois-tu que la grammaire te sera utile pour ce que tu veux faire plus tard comme travail?
1
2
3
4
5
6
7
Pas du tout utile

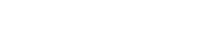
Très utile

10. Je crois qu'être bon en grammaire est...

$\begin{array}{lrlllrr}1 & 2 & 3 & 4 & 5 & 6 & 7 \\ \text { Pas du tout important } & & & & \text { Très important }\end{array}$

11. En comparaison avec les autres élèves, comment penses-tu réussir cette année en grammaire ?
1
2
3
4
56
7
Beaucoup moins bien que les autres élèves
Beaucoup mieux que les autres élèves

12. Est-ce que la quantité d'efforts nécessaire pour bien réussir les activités de grammaire les plus difficiles en vaut la peine?
1
2
3
4
5
6
7
Pas vraiment

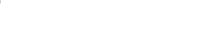

$\begin{array}{lll}5 & 6 \\ & \end{array}$

